\title{
Linx
}

Revue des linguistes de l'université Paris X Nanterre

64-65 | 2011

Les genres de discours vus par la grammaire

\section{Représenter les relations entre grammaire et genres de discours : l'exemple des commentaires sportifs}

\section{Mustapha Krazem}

\section{OpenEdition}

\section{Journals}

Édition électronique

URL : http://journals.openedition.org/linx/1400

DOI : 10.4000/linx.1400

ISSN : 2118-9692

Éditeur

Presses universitaires de Paris Nanterre

\section{Édition imprimée}

Date de publication : 1 juillet 2011

Pagination : 45-68

ISSN : 0246-8743

\section{Référence électronique}

Mustapha Krazem, «Représenter les relations entre grammaire et genres de discours : l'exemple des commentaires sportifs », Linx [En ligne], 64-65 | 2011, mis en ligne le 01 juillet 2014, consulté le 02 mai 2019. URL : http://journals.openedition.org/linx/1400 ; DOI : 10.4000/linx.1400 


\title{
Représenter les relations entre grammaire et genres de discours : l'exemple des commentaires sportifs ${ }^{1}$
}

\author{
Mustapha Krazem \\ CPTC/GRELISC Université de Bourgogne
}

\section{Introduction}

L'objectif de cet article est de proposer une représentation des genres de discours (au sens large) qui accorderait la primauté aux conditions d'apparition remarquables des éléments grammaticaux: haute fréquence, agrammaticalité locale, voire "néologie grammaticale». Cependant, représenter ne signifie pas classer. Nous savons qu'il est vain de classer les genres en utilisant les faits de grammaire. Petitjean (1989), Rastier (2001) ou Adam (2010) ont montré l'impasse où mènent de telles entreprises. Parler des "genres de l'imparfait » n'aboutirait qu'à dresser la liste hétérogène des genres où l'imparfait apparait.

Mais comment représenter les genres en accordant à la grammaire sa place légitime, surtout si on prend au sérieux Bakhtine lorsqu'il affirmait que la langue est apprise à travers les genres de discours? On devine depuis longtemps (BlancheBenveniste, 1999) qu'il existe des relations entre certains éléments de langue et les conditions «extérieures » où ils sont produits. Une explication commode serait

\footnotetext{
${ }^{1}$ Remerciements pour leurs remarques, critiques et suggestions au relecteur anonyme de la revue, à Olivia Guérin et Daria Toussaint.
} 
d'invoquer, en s'en contentant, le « contexte genre », bien que Kleiber (2009) mette en garde les études qui utilisent l'argument du contexte sans lui donner un statut rigoureux. Nous prétendrons ici à cette rigueur, en associant de la façon la plus insécable possible un élément linguistique à une réalité extérieure à la langue mais interne au genre de discours où il apparait, que cette réalité soit communicationnelle, culturelle, psychologique ou encore, plus matériellement, sonore, iconique, typographique.... C'est donc bien de faits dont il sera question ici, qu'ils soient linguistiques, communicationnels ou extralinguistiques.

Nous ne chercherons donc pas à classer ou à hiérarchiser mais à représenter les figures génériques (genres, genres inclus, sous-genres, « sous-sous » genres...) afin d'identifier des figures génériques minimales insécables (que nous appellerons des générèmes).

L'intérêt d'une représentation telle que nous la proposerons ici sera d'isoler de la façon la plus fine possible les facteurs qui influent sur l'apparition des éléments grammaticaux, afin de mieux en comprendre le fonctionnement. Nous ne nous intéresserons pas aux éléments de grammaire en tant qu'indicateurs stables d'un genre $\mathrm{x}$ mais nous favoriserons une utilisation du genre pour mieux expliquer l'élément pour lui-même. Le genre de discours, en se redéployant en générèmes, devient ainsi un terrain ciblé d'observation grammaticale. Ces générèmes, nous le verrons, n'ont qu'un intérêt relatif dans l'optique d'une approche culturelle des genres de discours. Toutefois, parce qu'ils seront générés dans le cadre d'une structure de traits, ils peuvent rendre compte non seulement de figures génériques et de sous-genres existants, mais aussi de figures génériques ayant existé ou potentielles, surtout lorsque celles-ci n'ont pas été dénommées. C'est là un aspect important de notre démarche : les classements, hiérarchiques ou non, habituellement proposés ne classent pas tous les objets discursifs (que nous appellerons figures génériques pour éviter des conflits définitoires), seulement ceux qui sont nommés. Cet aspect lexicologique est crucial. Nous insisterons sur le fait que le passage par les sous-genres, en tant que figures lexicalisées, est une étape préalable à l'identification des générèmes, parce les dénominations sont les premières entrées accessibles.

Notre modèle sera éprouvé sur les commentaires sportifs (dorénavant CS). Ce genre de discours, du moins dans ses aspects saillants, s'avère fertile à plus d'un titre. Il est perméable aux évolutions technologiques, lesquelles se poursuivent encore. C'est un genre ancien (la presse sportive a plus de cent ans). Le commentaire est multi canal : radio, télé, presse. Il a une prosodie remarquable (Mathon/Boulakia, 2011). Et surtout, les CS contiennent des formes linguistiques singulières dont l'analyse fine reste largement à effectuer (Deulofeu, 2000, Bres, 2007, Labeau, 2007, Krazem, 2005 et 2011). Le plus souvent, ces formes obéissent à une caractéristique majeure de ces commentaires : ils disent la relation au moment présent. Cela leur confère un statut d'observatoire privilégié, simple à attester empiriquement, pour étudier les rapports entre la langue et sa production, entre un événement et son énonciation.

Notre corpus sportif n'est donc ni exotique ni anecdotique. Il est autant pertinent par son ancrage en phase directe avec l'évolution de la société (autant culturelle que technologique) que par sa fécondité grammaticale.

Dans la première partie de notre contribution, nous nous emploierons à déplacer l'angle de vue posé habituellement sur les genres et les sous-genres de 
discours, catégories que nous tenons pour structuralement identiques. Nous introduirons la catégorie plus large de figure générique, qui permettra de pallier les carences du lexique. Nous proposerons une unité linguistique pratique, le géérème, qui facilitera la discrétisation de la face formelle (langue mais aussi image, son, etc.) des genres de discours au moyen d'une structure de traits.

Dans la seconde partie, nous présenterons les différentes composantes de la représentation linguistique des commentaires sportifs en insistant sur la relation entre des réalités externes (télé, radio, direct, différé, presse,...) et de nombreux faits de langue. Il en découlera une liste d'observables que nous convertirons en traits oppositifs.

Dans la troisième partie, nous présenterons la structure de traits caractérisant la globalité des CS. Puis nous la déploierons dans toutes ses combinaisons possibles, générant ainsi une liste exhaustive de générèmes associables à des types de commentaires sportifs réels, potentiels ou impossibles.

\section{Figures génériques :}

\section{Genre, Sous-Genre, Générème et Figures Genériques Intermédiaires}

Définir un genre de discours n'est pas chose aisée. Aussi, affirmons d'emblée ce que nous appellerons genre de discours et par transitivité, sous-genre, car parmi toutes les difficultés à cerner de façon précise et indiscutable la définition de ces objets discursifs, nous relevons une source d'ambiguité défavorable à une approche grammaticale des genres de discours : les genres de discours sont sujets à l'opposition type/occurrences de Kleiber (2009). Les CS sont susceptibles de deux approches, non contradictoires d'un point de vue social, mais perturbantes lorsqu'on souhaite être au plus près des appariements entre faits de langue et faits de externes. L'approche par occurrences favorise une approche textuelle tandis que l'approche par types prépare mieux une description linguistique interne des éléments de langue pris isolément. Soit donc le commentaire sportif «en direct télé». Ce sous-genre des CS, lorsqu'il est étudié par le biais de ses occurrences, est hétérogène. Il contient des séquences de commentaires d'actions plus (A) ou moins (B) rapides, des rediffusions courtes d'actions à vitesse normale $(\mathrm{C})$ ou ralentie $(\mathrm{D})$, des descriptions d'ambiance (E), des dialogues entre les commentateurs (F), des annonces diverses, par exemple un autre RV télé $(\mathrm{G})$, des questions pour un jeu concours $(\mathrm{H})$, des interviews, principalement à la mi-temps (I) mais pas seulement (J), des narrations d'événements récents qui intéressent le téléspectateur, par exemple les suites d'une affaire disciplinaires concernant un joueur $(\mathrm{K})$ et nous en oublions certainement $(\mathrm{L})$.

Le CS vu par ses occurrences est particulièrement compatible avec l'analyse par séquences. Les liaisons, sutures, renvois entre les séquences montreraient rapidement que celles-ci ne se combinent pas par une simple addition technique et mécanique. Elle contribue à créer un effet de généricité tout en dégageant des propriétés linguistiques fines (Guérin, 2011). Cependant, l'approche par occurrences n'est pas la seule possible. En effet, elle oblige à considérer des séquences qui ne sont pas réellement tenues pour des CS, ce qui risque de noyer la précision des observations sur les faits de langue, exception faite des mécanismes linguistiques inhérents à la progression textuelle et à 
l'imbrication des séquences. L'approche par types ne va considérer que les séquences A, B C et D (éventuellement E) sans tenir compte de F, G, H, I, J, K ou L.

L'opposition type/occurrence appliquée aux commentaires sportifs est analogue à une métonymie. D'un côté le genre de discours dans ses manifestations réelles et complètes, de l'autre le genre de discours limité à ses manifestations saillantes justifiant sa dénomination lexicale ${ }^{2}$. Car c'est un fait remarquable : bien souvent, l'appellation des genres de discours ne retient que le type. C'est à cause des séquences $\mathrm{A}, \mathrm{B}, \mathrm{C}$ ou $\mathrm{D}$ que les CS sont appelés "commentaires sportifs", même si les occurrences de ces séquences n'apparaissent quasiment jamais isolément. L'ensemble de ces séquences typiques des CS est constitué de ce que nous appellerons des figures génériques. Celles-ci amplifient la dimension «biface » (interne/linguistique et externe/social) des genres de discours (Branca, 1999), en englobant tous les types de configurations discursives associant une fonction communicationnelle, sociale et/culturelle (le "signifié ») à un ensemble solidaire de formes linguistiques ou non (le « signifiant »), lequel « signifiant » fera une large place aux éléments non linguistiques (écrit, image, son, photo, schéma, tableau,...) car le genre CS est notoirement multimodal (Martin et Rose, 2010). Cela influence grandement les formes linguistiques convoquées même si la langue naturelle ${ }^{3}$ est le principal vecteur de la signification des CS.

C'est donc en tant que figures génériques que nous étudierons les commentaires sportifs, qu'ils soient genre, sous-genre et nous le verrons, générème ou genre intermédiaire.

\subsection{Du genre au sous-genre}

Les débats se proposant de définir les genres de discours ont été nombreux, mais on ne s'attarde pas suffisamment sur la définition du sous-genre, comme si définir ce qui est présenté généralement comme un étage inférieur découle bien naturellement de la définition de la catégorie qui chapeaute. Or, la définition des sousgenres est une entreprise bien plus ardue qu'il n'y parait. En premier lieu, les difficultés définitionnelles des GD sont transmises automatiquement en l'état sur ses sousgenres. L'opération de sous-détermination reporte les problèmes sans les évacuer. En second lieu, le sous-genre est loin d'être « un » niveau hiérarchique unique. Il est luimême constitué de sous-genres. Ainsi le genre "petites annonces» contient-il les «annonces d'emploi », lesquelles comprennent les «demandes» et les «offres». Le fonctionnement est comparable à celui des poupées russes, parce qu'une structure commune à tous les objets se maintient, objets qui s'incluent dans un mouvement qui

\footnotetext{
${ }^{2}$ Le choix du type a un coût : il faut normaliser, identifier une structure canonique à chaque figure générique. Certes, nous suivons Maingueneau (2004) lorsqu'il classe les genres en fonction de leur degré de contraintes (des routines aux genres littéraires), mais sans renoncer à stabiliser les formes linguistiques employées, même si elles sont sujettes à des violations assumées. Les genres gagnent à être stabilisés autant que possible pour que « l'air de famille » d'Adam et Heidmann (2009) devienne, au moins le temps de l'analyse, une "photo de famille».

${ }^{3}$ Si certains genres utilisent la langue de façon minoritaire, par exemple les affiches publicitaires, peut-on imaginer des genres de discours sans langue naturelle? La question n'est pas triviale. La poésie contemporaine s'est donné l'autorisation d'oublier la langue si besoin (Donguy, 2007).
} 
aboutit à une plus grande précision à la fois de la forme («le signifiant») et de la fonction communicationnelle, culturelle et/ou sociale («le signifié »).

Or, si on considère pleinement cette succession dont les étapes ne sont pas limitées, l'opposition entre genre et sous-genre devient quasiment caduque. Le genre est seulement le point de départ d'une chaine qui s'achève par des productions discursives structurées parfois étiquetées «sous-genres ». La relation genre/sous-genre n'est pas une relation entre un objet $\mathrm{A}$ et d'autres $\{\mathrm{a}, \mathrm{b}, \mathrm{c} \ldots \mathrm{n}\}$ de nature différente, les seconds concrétisant le premier. C'est une relation hiérarchique d'emboîtement entre des objets de nature similaire.

Nous insistons sur l'adverbe "parfois » parce que la division genre/sous-genre, prise dans la totalité de ses manifestations, n'est pas une organisation hiérarchique du monde réel des productions discursives structurées. C'est une hiérarchisation hyper/hyponymique des dénominations existantes de ces dernières. Or, l'hyperonymie n'est pas une division du monde mais une division du lexique, lequel, on le sait, dénomme de façon «imparfaite/aléatoire/conjoncturelle » le monde réel, en fonction de l'aspect communicationnel/social/culturel sélectionné par les langues et les cultures considérées.

Nous retrouvons cette «imperfection» dans la relation genre/sous-genre lorsqu'il s'agit d'y privilégier la grammaire : certains genres étiquetés par la langue n'ont pas ou peu de pertinence linguistique alors que l'opposition communicationnelle ou culturelle des sous-genres est légitime. Il en va ainsi de la tragédie vs la comédie, tandis que bien des sous-genres contiennent en réalité des sortes de "sous-sous-genres» distinguables par des éléments de langue sans pour autant être eux-mêmes dénommés. Toutefois, et ce point est crucial, la perception des genres de discours est fortement liée à leur dénomination. Nous y reviendrons dans la deuxième partie.

\subsection{Vers une arborescence générique grammaticale}

Pour atteindre une précision grammaticale aussi fine que possible, les seuls genres/sous-genres ayant une entrée dans le dictionnaire ou bien lexicalisés par composition ne sont pas suffisants. Une arborescence dont la tête serait le genre et dont les ramifications iraient jusqu'à des productions dont la granularité grammaticale serait insécable proposera presque immanquablement des nœuds lacunaires. La figure (1) ci-dessous nous propose une représentation prototypique d'une arborescence générique privilégiant les spécificités grammaticales. A partir d'un Genre de Discours source (GD), étiqueté lexicalement, se déploie une arborescence dont les branches terminales aboutissent à des configurations structurées discursives insécables linguistiquement. Les nœuds sont occupés par des Figures Génériques de natures différentes. Ce sont des Sous-Genres (SG) lorsque ceux-ci sont étiquetés lexicalement, des Figures Génériques Intermédiaires (FGI) lorsque ceux-ci ne sont pas étiquetés lexicalement et qu'ils peuvent

\footnotetext{
${ }^{4}$ Le « point de départ » est davantage une nécessité méthodologique qu'une affirmation de principe. Bien souvent, un genre peut devenir le sous-genre d'un autre sans que la chaîne ne s'achève vraiment ou en associant des genres qui ont assez peu de points communs. Avons-nous beaucoup d'intérêts à tenir les règles de jeu pour un sous-genre des modes d'emploi ? et les modes d'emploi un sous-genre des notices?
} 
encore être sous-divisés en configurations discursives plus fines. Ces subdivisions dominent dans les branches terminales, soit des Générèmes (Gn) soit des SG lorsque ces derniers sont insécables, ce qui les assimilent alors à des générèmes ${ }^{5}$.

(1) Arborescence d'un genre de discours

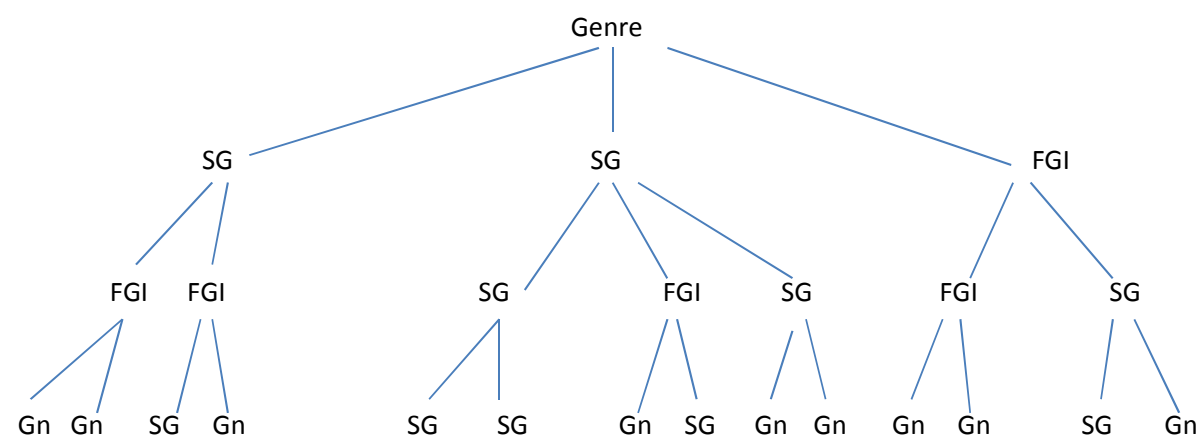

\subsection{Diagramme des figures génériques}

En recourant aux générèmes, nous visons à diviser le monde discursif réel (donc présent et passé) et potentiel de sorte que la moindre différence régulière d'un fait de langue ${ }^{6}$ entraine un générème différent. Ainsi, l'interprétation des sujets (dont le sujet vide) fait de l'offre d'emploi un générème différent de la demande d'emploi.

Cependant, à quoi ressemblent formellement les articulations entre les faits de grammaire et ce que nous appellerons « faits externes », bien que ceux-ci, appartiennent à la figure générique (genre, sous-genre etc.) ?

- Elles peuvent provenir de ce que nous avons appelé par commodité le « signifié » des figures génériques: situation de communication, fonction de communication, domaine culturel, stratégie énonciative, dispositif énonciatif, convention sociale, etc. C'est ainsi que dans les CS les pronoms utilisés pour désigner les auditeurs/ téléspectateurs sont très contraints et peu marqués morphologiquement (surtout quand il y a deux commentateurs).

- Elles peuvent provenir du complémentaire du «signifiant»: schéma, photo, film, son, écrit, typographie, calligraphie, médias, technologie, gestuel, etc. C’est ainsi que la précision des actants est moins requise lorsque les commentaires sont visuels.

\footnotetext{
${ }^{5}$ Cela n'est pas exclu non plus pour un Genre de Discours si celui-ci n’a qu'une seule concrétude. Nous n'avons pas trouvé d'exemples vraiment convaincants. Peut-être les mises en garde sur les paquets de cigarettes?

${ }^{6} \mathrm{Un}$ ou plusieurs faits liés à condition qu'ils soient eux-mêmes insécables dans l'ensemble du générème considéré.
} 
Le diagramme (2) ci-dessous représente les relations des faits externes vers les faits de langues génériques ${ }^{7}$. Elle n'est pas bijective puisqu'un fait de grammaire peut résulter de plusieurs faits externes: la haute fréquence dans les CS des phrases averbales se réduisant au seul nom propre est due à la fois au visuel et au direct. Inversement, un fait externe provoque au moins un fait de grammaire. C'est particulièrement vrai pour la situation « en direct », qui entraîne, outre les interjections, l'absence d'élision du sujet ou encore les noms propres seuls.

Le diagramme (2) ne considère que les seuls éléments susceptibles d'être mis en relation avec un fait de grammaire marqué génériquement. Le paramètre «image en couleur» ne serait pas un fait externe «impactant $»^{8}$ car il n'a aucune incidence linguistique ${ }^{9}$. De même, le type de journal (presse d'opinion/presse d'information) n'a pas davantage d'incidence.

Sont donc exclus de la caractérisation des figures : les éléments grammaticaux jamais génériques, les éléments susceptibles d’être génériques non concernés par le genre étudié ${ }^{10}$, les paramètres formels ou communicationnels ${ }^{11}$ non pertinents. Sont également exclues (à ce stade de l'analyse) les relations entre les signifiés et les signifiants non linguistiques qui relèvent d'autres logiques sémiotiques.

Formellement, le diagramme des genres de discours contient celui des sousgenres et surtout des générèmes. Le principe est d'affiner les diagrammes appartenant au CS de sorte qu'ils soient les plus spécifiés possibles, ce qui implique que ces diagrammes deviennent insécables. Ils gardent tous néanmoins les relations communes à tous les CS, distinguées par les flèches visibles. Le genre de discours d'origine a un diagramme plus englobant que les Sous-Genres ou les Genres Intermédiaires. Les générèmes proposent des diagrammes non englobants, qui se distinguent des autres générèmes sur une seule relation. Nous verrons, à l'épreuve des CS, que cela implique la construction ad hoc de tous les générèmes prévus par l'activation d'une structure de traits (\$3.3), y compris des générèmes impossibles.

\footnotetext{
${ }^{7}$ Un fait de langue générique comprend à la fois la nature du fait, par exemple «interjection oh» mais aussi sa fréquence. Dans les CS, l'interjection «oh» recouvre trois éléments : oh exclu (accompli), oh fréquent (direct pur), oh rare (replay/ralenti).

${ }^{8}$ Nous réservons ce néologisme récent à l'ensemble des faits influant sur la grammaire.

${ }^{9}$ Notons toutefois que du temps de la TV noir et blanc, cet élément était intégré par les commentateurs.

${ }^{10}$ Le gérondif n'a par exemple pas le même rendement générique dans les didascalies.

${ }^{11} \mathrm{La}$ couleur politique d'un journal ou bien l'appartenance ou non d'une télé au service public n'a pas d'incidence sur les CS.
} 
(2) Diagramme de la relation des faits externes vers les faits de langue

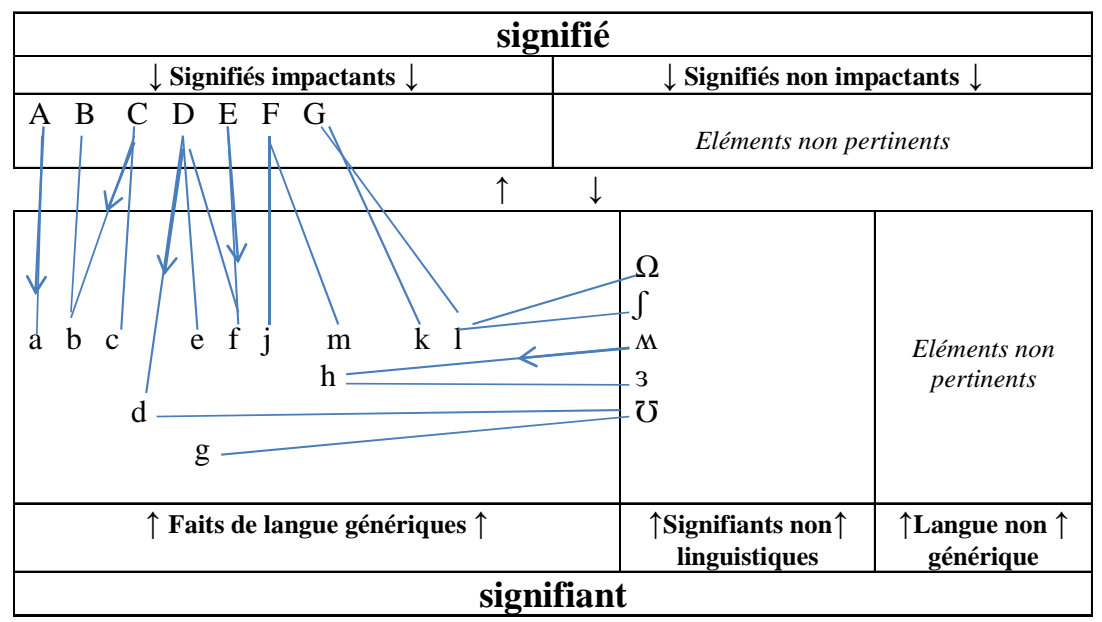

\subsection{Bilan}

En isolant les configurations discursives jusqu'au niveau où un seul élément linguistique (ou groupe d'éléments linguistiques solidaires insécables) varie, les générèmes se présentent comme des unités stables, opposables entre elles, pour lesquelles une analyse de type signifiant/signifié devient possible. Il importe peu que ces générèmes soient ou non de réels signes linguistiques crédibles, ou seulement des unités pratiques (Deulofeu, 2000, Adam, 1999). Le tableau (3) récapitule les différences de statut entre Genres de Discours(GD), Sous-Genres (SG), Genres Intermédiaires (GI) et Générème (Gn). Nous précisons en note ce que nous entendons par méronymie ${ }^{12}$ et variation ${ }^{13}$.

\footnotetext{
12 Certes un genre de discours peut s'inclure dans un genre de discours pour créer une scénographie particulière. Mais nous préférons réserver un sens plus strict à la méronymie, celui de partie constitutive d'un tout qui l'englobe. Ainsi les titres sont-ils des méronymes. Si on les tient pour des sous-genres (ou genres inclus pour Rastier, 2001) alors un sous-genre peut être méronyme. Plusieurs générèmes des CS n'existent qu'en tant que méronyme d'une autre configuration discursive (3.3).

${ }^{13}$ Nous pensons que les violations individuelles partent de la conscience du locuteur d'être face à un genre identifié. Aussi, ce que le locuteur croit violer est une configuration lexicalisée (genre ou sousgenre) même si en définitive il ne viole qu'un générème ou un genre intermédiaire.

Reste la question des variations collectives envers un genre. Par exemple l'emploi du futur de l'indicatif dans les didascalies (Krazem, 2004). Si elles sont liées régulièrement à un paramètre externe (pour les didascalies affirmation de l'omniscience de l'auteur) alors elles construisent un générème. Ces variations collectives conscientes constituent une partie du champ de la grammaire seconde de Blanche-Benveniste.
} 
(3) Bilan des figures génériques

\begin{tabular}{|c|c|c|c|c|}
\hline & $\begin{array}{l}\text { GENRE DE } \\
\text { DISCOURS }\end{array}$ & $\begin{array}{l}\text { SOUS- } \\
\text { GENRE }\end{array}$ & GENEREME & $\begin{array}{l}\text { FIGURE } \\
\text { GENERIQUE } \\
\text { INTERMEDIAIRE }\end{array}$ \\
\hline $\begin{array}{l}\text { Unité } \\
\text { existante ou } \\
\text { ayant existé }\end{array}$ & toujours & Toujours & partiellement & partiellement \\
\hline $\begin{array}{l}\text { Existence sans } \\
\text { dénomination } \\
\text { lexicale }\end{array}$ & non & non & Oui ou non & toujours \\
\hline $\begin{array}{l}\text { Potentiel- } \\
\text { lement } \\
\text { existant }\end{array}$ & $\begin{array}{l}\text { Non sauf } \\
\text { imaginaire }^{14}\end{array}$ & non & éventuellement & éventuellement \\
\hline $\begin{array}{l}\text { Potentiel- } \\
\text { lement } \\
\text { impossible }\end{array}$ & non & non & Eventuellement & Eventuellement \\
\hline $\begin{array}{l}\text { Peut se } \\
\text { subdiviser }\end{array}$ & $\begin{array}{l}\text { Oui sauf si } \\
\text { concrétisation } \\
\text { unique } \\
\text { (=générème) }\end{array}$ & $\begin{array}{l}\text { Oui, sauf si } \\
\text { le sous-genre } \\
\text { est un } \\
\text { générème }\end{array}$ & $\begin{array}{l}\text { Impossible } \\
\text { (contradictoire) }\end{array}$ & $\begin{array}{l}\text { Oui, } \\
\text { obligatoirement }\end{array}$ \\
\hline $\begin{array}{l}\text { Concrétise un } \\
\text { Genre de } \\
\text { discours }\end{array}$ & $\begin{array}{l}\text { Non, à part } \\
\text { lui-même }\end{array}$ & oui & Oui & Oui \\
\hline $\begin{array}{l}\text { Aptitude à la } \\
\text { méronymie }\end{array}$ & $\begin{array}{l}\text { Non, pas } \\
\text { naturellement }\end{array}$ & $\begin{array}{l}\text { Oui/Non, } \\
\text { selon } \\
\text { théories }\end{array}$ & $\begin{array}{l}\text { Oui pour } \\
\text { certains }\end{array}$ & Oui pour certains \\
\hline $\begin{array}{l}\text { Variation } \\
\text { individuelle }\end{array}$ & oui & oui & $\begin{array}{l}\text { Oui si sous- } \\
\text { genre }\end{array}$ & non \\
\hline
\end{tabular}

\section{Mise en place d'une représentation grammaticale}

Revenons sur un problème annoncé plus haut: la perception intellectuelle des genres de discours (ou des sous-genres) passe par leur dénomination. Puisque les générèmes prétendent au plus fin degré de granularité linguistique, il est nécessaire pour les repérer d'explorer d'abord la construction formelle des genres/ sous-genres que le lexique fait exister. En effet, la délimitation des paramètres externes ne peut s'effectuer intuitivement que sur les propriétés saillantes des sous-genres, même si, comme nous le verrons (\$3.3), certains paramètres leur échappent. Nous admettons ainsi que les sous-genres, outre une spécialisation communicationnelle ou culturelle plus précise que le genre, prennent acte aussi de variations formelles plus fines. Aussi avons-nous d'abord défriché la construction linguistique des CS à partir de six sous-

\footnotetext{
${ }^{14}$ Les auteurs de science-fiction ont déjà «inventé » des genres à travers des supports imaginés/ anticipés. Par exemple le téléphone avec image du correspondant est un rêve ancien qui s'est concrétisé. Nous doutons -mais pourquoi pas après tout- que la science-fiction anticipait aussi les sous-genres....
} 
genres sportifs : TV direct et différé, radio direct et différé, internet en direct et presse écrite. Hormis le commentaire internet, chacun en connait l'existence (au moins lexicalement), y compris les locuteurs non-amateurs. Le commentaire par internet néanmoins commence à être connu et désigné lexicalement. Nous considérons, dans une perspective diachronique, qu'il s'agit d'un sous-genre (SG), encore émergent.

Il résulte de cette étude des sous-genres qu'il convient de distinguer trois composantes a priori autonomes (mais pas indépendantes) :

- La fonction communicative (ou dispositif énonciatif) ${ }^{15}$. Nous retrouverons là ce qui unifie l'ensemble des commentaires sportifs, ce qui rend saillant et justifie le genre « commentaire sportif»

- Le lexique, important pour les CS. Il contribue à justifier la dénomination commentaires sportifs.

- les paramètres externes provoquant des faits de grammaire et construisant par combinaisons, les générèmes et figures génériques intermédiaires.

\subsection{La fonction communicative ou sociale : le commentaire d'événement}

Les faits de langue peuvent se regrouper pour structurer une fonction communicative, sociale ou culturelle ${ }^{16}$. Cette fonction doit être rendue formalisable à l'aide d'un ensemble fini de traits linguistiques, indépendamment d'un genre de discours singulier ${ }^{17}$. Nous nous attardons ici sur la fonction «commentaire d'événement», de sorte qu'elle soit applicable, avant de la spécialiser à tous les commentaires: un événement sportif, un mariage princier, une pièce de théâtre (didascalies), des débats parlementaires, des itinéraires de randonnée ${ }^{18} \ldots$ Le commentaire est donc un discours décrivant un événement concomitant ou non à l'énonciation produite par un énonciateur en direction d'un allocutaire. Ce que nous formaliserons par (4).

(4) $\operatorname{En}(\mathrm{T} 1):[\mathrm{L} \quad \underline{\mathrm{Ev}(\mathrm{Ta})} \rightarrow 1]$

L est l'énonciateur dans la situation d'énonciation (En). Il est nécessairement associé à un allocutaire 1 . L'énonciateur L, à un moment T1, décrit un événement (Ev)

\footnotetext{
15 Un point théorique important cependant : les facteurs de variation tels qu'ils sont inscrits dans la fonction communicative (ou dispositif énonciatif) sont-ils internes ou externes à la langue ? Nous pensons pour notre part qu'ils sont (majoritairement) externes. Ce sont des considérations externes qui font que « je » emploie « je » et non « tu» ou un impératif plutôt qu'un infinitif de prescription. L'enjeu est important car il affecte fortement les relations entre genre et grammaire. Nous ne développerons pas ici cette prise de position.

${ }^{16}$ Ce regroupement s'observe aussi de façon «naturelle » sur la dénomination lexicale des GD, voir présentation du numéro.

${ }^{17}$ Cette approche contient des points communs avec celle d'Adam (2001) pour les genres procéduraux. Elle correspond partiellement aux dimensions de Biber (1988). Ces groupes de faits n'apparaissent cependant pas forcément au même stade de l'analyse. Notre approche part de catégories communicationnelles existantes. Les « activités discursives » de Kerbrat et Traverso (2004) se prêteraient davantage à ce mode de formalisation.

18 Adam classe lui les itinéraires de randonnée dans les genres procéduraux.
} 
qui se situe dans un moment Ta. Le sens de la flèche institue une relation unilatérale ( $a$ priori pas de feed back). L'événement Ev peut être réel, potentiel ou fictionnel. Tel que nous l'entendons ici, Ev suppose une durée, ce qui implique des successions de procès. Le commentateur n'agit pas sur l'événement. Enfin, le moment de l'énonciation T1 est ou n'est pas le même que le moment TA de l'événement.

Appliquée aux commentaires sportifs, cette configuration est concrétisée comme suit :

- L'événement sportif implique un langage spécifique (voir plus loin). Ajoutons rapidement que les sports contiennent chacun des singularités influant à la marge la grammaire du commentaire (vitesse/nombre de participants, sports d'opposition ou individuels...)

- Le temps de l'énonciation est ou n'est pas contemporain du temps de l'événement. Nous retrouvons là l'opposition direct/différé.

- Le commentateur est généralement spécialisé. C'est un professionnel. Certaines tournures seraient analysables par l'intermédiaire de la "grammaire seconde» (Deulofeu, 2000). La spécialisation des journalistes connait toutefois des limites avec les sports peu médiatisés dont certains ne sont visibles en direct (du moins sur les chaînes gratuites) que lors des jeux olympiques (aviron, lutte, curling...). Pour être complet, ajoutons que s'expriment très fréquemment des «consultants» (particulièrement utiles pour les sports peu médiatisés) qui participent ponctuellement aux commentaires. Leur langue est moins typée par le genre de discours ${ }^{19}$. Cependant, leur présence construit une situation trilogique (Kerbrat, 1995) qui n'existait pas antérieurement ${ }^{20}$.

- L'allocutaire 1 est toujours «pluriel ». Le journaliste s'adresse à une multitude de personnes avec lesquelles aucun retour n'est envisagé. Néanmoins, depuis quelque temps, une forme d'interaction émerge à travers les sms ou les réseaux sociaux. De plus, le public est généralement spécialisé. Il maittrise le lexique et les règles des sports dont il écoute les commentaires. C'est particulièrement vrai des sports les plus retransmis (foot, rugby, cyclisme, tennis). Nous ne suivons donc pas Mathon et Boulakia (2011) lorsqu'ils parlent de locuteurs «virtuels». Certes ils sont absents physiquement mais ils diffèrent nettement de l'allocutaire des didascalies ou des guides de randonnées. Il est assez fréquent que les commentateurs utilisent des adresses aux téléspectateurs/auditeurs.

Le module commentaire est donc très contraint dans les CS. Mais tous les commentaires d'événement n'ont pas le même dispositif communicationnel. Le commentaire didascalique est partiellement distinct [-interaction, + Allocutaire unique, +Enonciateur [+neutre], -Evénement réel]. Cela entraîne des différences linguistiques

\footnotetext{
${ }^{19}$ Dans certaines diffusions télévisées, depuis quelque temps, une troisième intervenant est sollicité. Il joue le rôle de fouineur ou d'intervieweur pendant les mi-temps.

${ }^{20}$ Nous avons cependant négligé ce point dans notre structure de traits générale des CS ( $\$ 3$ afin d'éviter une complexité supplémentaire. Toutefois, la prise en compte du consultant introduit un positionnement énonciatif différant du commentateur des générèmes [[-écrit] [+direct]].
} 
avec les CS, notamment dans l'emploi des pronoms personnels. L'emploi fréquent et large du pronom « on » dans les didascalies en est une illustration ${ }^{21}$.

\subsection{La composante lexicale}

Intuitivement, on s'attend à ce que la composante lexicale contribue prioritairement à la reconnaissance des GD par les locuteurs. Ce n'est pas toujours avéré. Les didascalies ne se distinguent par aucun vocabulaire particulier, pas même le langage technique de la mise en scène. De plus, un lexique générique peut connaitre des degrés de spécialisation, pour participer par exemple à des paliers plus ou moins élevés de vulgarisation. Cela spécifie des GD opposables (article scientifique/article journalistique scientifique).

Le vocabulaire sportif varie d'un sport à l'autre. Une classification grossière nous conduit à distinguer :

- les termes qui n'existent que dans le domaine sportif : salto, ace, corner, uppercut, break, Kovacs

- les nominalisations déverbales avec un sens spécifique ou exclusif : tir, relance, centre, contre, sprint, drop, shoot...)

- les noms prédicatifs. Ces termes désignent certes un objet mais, dans certaines conditions, peuvent être interprétés comme processus ou résultat de processus : but, un mur, carton, ballon,... Un « long ballon » n'est pas un ballon de forme longue mais une passe effectuée à grande distance.

Les relations entre le lexique et les genres de discours ne s'anticipent donc pas d'emblée et montrent parfois des interactions avec les faits de grammaire génériques. Le lexique sportif participe à sa façon à la grammaire des CS car il en accompagne une propriété forte : suivre la vitesse de l'événement. L'exemple (5) correspond à une séquence à la barre fixe (gymnastique JO de Pékin). La séquence étant courte, aller à l'essentiel est impératif.

(5) allez c'est parti / prise d'élan demi-tour à l'équilibre tout de suite et là ça accélère immédiatement pour un lâcher de barre / Kovacs plus vrille et un salto pardessus la barre avec une vrille / on sent qu'il est parti avec une grosse envie/ beaucoup de rythme / dislocation / franchissement dorsale de barre tendu / figure du pigeon / tour volant plus demi-tour / Passage sur le dos et quatre tours / Il est sorti/

\subsection{Les paramètres externes pertinents}

Les faits linguistiques sujets à variation sont nombreux, au point que les CS suscitent depuis longtemps un intérêt scientifique, surtout par leur rapport au présent physique. Le commentaire en direct est un passage quasi obligé pour décrire le présent

\footnotetext{
${ }^{21}$ Notre approche croise celle de Bouquet (2007) : ce ne sont pas les mêmes valeurs de « on » qui sont attestées dans les deux types de commentaire. Dans le CS, «on» est moins fréquent et soit équivalent à «nous» soit (valeur générique plus spécifique) un mode d'investissement englobant l'auditeur : On est maintenant à une quarantaine de mètres des buts de Marseille.
} 
de l'indicatif ${ }^{22}$. De son côté, le commentaire écrit a retenu l'attention (Labeau, 2007) à cause de l'utilisation originale du passé simple. Le CS est également un des genres dans lequel on rencontre le plus d'énoncés averbaux, énoncés dont on sait qu'ils ont un rapport étroit avec le moment de l'énonciation.

\subsubsection{La temporalité : nécessité de distinguer les présents}

L'étude des formes temporelles est particulièrement féconde quand il s'agit de distinguer les différents types de commentaires. La relation avec le présent de l'événement constitue la principale contrainte qui structure la temporalité. Le présent psychologique, le présent physique et le présent linguistiques (Wilmet, 1980) ont une grande importance dans les commentaires en direct (Krazem, 2005). D'autre part, la durée de l'énonciation interfère sur la syntaxe et la temporalité. La trace la plus originale en est l'allongement des voyelles, qui miment la durée d'une action. Dans l'exemple typique construit (6), l'allongement sur le «en » correspond à la course du ballon ${ }^{23}$.

(6) Et Zidane qui cennnnntre

Nous avons été conduit à intégrer dans la représentation par traits ce que nous appelons le "présent psychologique étendu » (Krazem, 2005), présent distinct du présent psychologique de Wilmet parce que le locuteur a un léger recul qui lui permet une posture juste en aval de l'événement, sans l'avoir pleinement analysé. La durée imputable au recul se cristallise sur deux facteurs :

(i) Pendant le temps nécessaire à l'énonciation, d'autres actions pertinentes sont possibles $^{24}$ (Bronckart, 2008 insiste sur la « durée de production » par opposition avec le «moment » de production);

(ii) La prise en compte par le journaliste des allocutaires, qui sont dans une situation de direct « repoussé » dans le commentaire en léger différé (Krazem, 2005).

\subsubsection{Faits de langue génériques dans les sous-genres ${ }^{25}$}

Le tableau (7) récapitule les tendances nettes observées dans les différents sousgenres. Le signe "+ » note une réalisation forte, «++» une réalisation très forte, comparativement à d'autres sous-genres sur le même point, le signe «- » une absence remarquable. L'absence de notation traduit une apparition non significative.

\footnotetext{
${ }^{22}$ Voir Krazem (2005) pour un état partiel de la question.

23 Nous renvoyons à Mathon et Boulakia (2011) pour une analyse plus fine de la prosodie des CS télé en direct.

${ }^{24}$ C'est particulièrement vrai à la radio. On s'en aperçoit quand on écoute un commentaire radio en regardant les images en direct, encore que depuis la TNT il devient difficile de remarquer ce fait artisanalement car quelques secondes de décalage séparent les deux médias.

25 Outre des travaux en cours ou non publiés, on trouvera des développements justifiant ce tableau dans Krazem (2005, 2009, 2011, 2012), Grinshpun et Krazem (2005), Labeau (2007), Mathon et Boulakia (2011), Deulofeu (2000), Brès (2007). Ce tableau n'intègre pas d'autres faits (connecteurs " mais », effet de chaine avec « et », passifs) insuffisamment étudiés à ce jour.
} 
(7) Tableau des faits de langue génériques des sous-genres lexicalisés

\begin{tabular}{|l|c|c|c|c|c|c|}
\hline & $\begin{array}{l}\text { TV } \\
\text { DIR }\end{array}$ & TV dif & $\begin{array}{l}\text { Radio } \\
\text { DIR }\end{array}$ & $\begin{array}{l}\text { Radio } \\
\text { dif }\end{array}$ & Presse & internet \\
\hline Phrase averbale complète & + & ++ & ++ & ++ & + & + \\
\hline GN, qui & + & + & ++ & + & - & - \\
\hline Nom propre seul & ++ & + & + & + & - & - \\
\hline Oh! & ++ & + & ++ & & - & - \\
\hline Avec X, qui & + & & + & & - & - \\
\hline Présent actuel & + & ++ & + & ++ & - & + \\
\hline Présent historique & - & & - & & + & \\
\hline Passé simple & - & - & - & - & + & - \\
\hline Futur (ultérieur) & - & & - & & & \\
\hline Futur historique & + & & + & + & + & \\
\hline Aller + inf (futur) & ++ & + & ++ & & - & \\
\hline Infinitif libre & - & - & - & - & + & \\
\hline Adresse allocutaire & + & + & & & & - \\
\hline Elision du sujet & - & + & - & + & + & + \\
\hline Allongement vocalique & + & - & ++ & + & - & - \\
\hline
\end{tabular}

\section{Représentation des commentaires sportifs : des sous-genres aux générèmes}

À partir de ce qui précède, nous sommes en mesure de proposer une représentation grammaticale des genres de discours. Nous formaliserons ces derniers à l'aide d'une structure de traits binaires. Après avoir exposé la méthodologie générale de construction de cette structure de traits, nous présenterons la structure générale correspondant au GD «commentaires sportifs». Enfin, nous redéploierons cette structure complète en isolant chaque générème impliqué par la combinaison exhaustive des traits oppositifs afin d'en donner les manifestations concrètes ou virtuelles. Au passage, nous affinerons les traits qui le nécessiteront.

\subsection{Méthodologie générale}

Les traits binaires sont présents dans chacun des trois composants du genre sportif. Cependant, seul le composant des paramètres externes est susceptible de variations significatives. Chaque trait correspond à une propriété non directement linguistique du genre de discours. Ces traits, non hiérarchisés, se combinent et s'associent aux composants fixes du GD (dispositif du commentaire et lexique) pour former une valeur terminale qui sera traduite en générème ${ }^{26}$. Quant au composant des paramètres externes, il est directement issu de l'observation des sous-genres. Le

${ }^{26}$ Notre approche a ceci de commun avec Bouquet (2007) qu'elle permet de structurer hiérarchiquement des valeurs terminales, donc directement observables sur du matériel linguistique et des réalisations génériques. 
tableau (8) s'emploie à établir une correspondance entre les sous-genres, leur perception intuitive et une identification des paramètres externes qui sont convertis en traits. L'opération technique consiste à convertir les éléments saillants en traits oppositifs (+/-écrit, +/-visuel, etc.) de sorte qu'une antonymie la plus totale possible soit opératoire. C'est ainsi que la télé ne «s'oppose » pas à la radio car elles ont en commun le canal oral, mais il en va autrement de + /-écrit (un commentaire est dit par la voix ou par l'écrit) ${ }^{27}$ ou +/-visuel (l'auditeur voit ou ne voit pas l'événement) ${ }^{28}$.

(8) Identification de paramètres binaires

\begin{tabular}{|l|l|l|}
\hline Sous-Genres & Perception intuitive & Paramètres saillants \\
\hline TV directe & image + son + direct & image/pas écrit/présent physique \\
\hline$T V$ différée & image + son + direct & image/pas écrit/présent psy étendu \\
\hline Radio directe & son + direct & pas d'image/pas écrit//présent physique \\
\hline Radio différée & son + différé & pas d'image/pas d'écrit/présent psy étendu \\
\hline Presse & écrit + nettement différé & écrit/événement accompli \\
\hline Internet & écrit + direct ou différé & écrit /événement pas accompli \\
\hline
\end{tabular}

Certains traits gagnent à être représentés en modules regroupant plusieurs traits. C'est principalement le cas ici du module «commentaire » que nous avons décrit plus haut. Il allège la représentation en mettant en commun les invariants de tous les générèmes, éventuellement en y insérant des variables minimes. Les commentaires sportifs, quels qu'ils soient, construisent le module commentaire de la façon suivante : [-Interaction, +Allocutaires [+multiples +spécialisés], +Enonciateur professionnel, +Evénement réel]. Ce module correspond aux relations « fléchées » du diagramme (2).

Notons toutefois que, la langue ne se laissant pas toujours saisir (faut-il dire «hélas »?) par la binarité, nous procéderons parfois à des ajustements techniques, principalement avec le présent direct et le présent psychologique étendu, qui ne sont pas compatibles. Cela ne poserait aucun problème sans les commentaires nettement décalés du moment présent. Le système d'opposition est plus fin. Le direct s'oppose au non-direct, lequel peut être +accompli (nettement accompli) ou -accompli (présent psychologique étendu) alors que le direct soit reste direct (dans ce cas nous le notons [+direct'] pour confirmer le trait dans le présent physique) soit est +accompli, comme nous le verrons concrètement plus loin. En effet, certains générèmes proposent des effets de direct bien qu'énoncés dans une situation nettement accomplie.

\subsection{Déploiement de la structure de traits}

Dans la structure de traits générale (9), les fonctions et les composantes sont notées en italiques. Ces italiques indiquent des ensembles de faits obligatoires, a priori plutôt contraints. Les traits binaires sont en minuscules. Lorsqu'ils sont déjà assignés,

\footnotetext{
27 Précisons néanmoins qu'il existe des émissions où un traducteur en langue des signes apparaît à l'écran.

${ }^{28}$ Il peut cependant entendre ou ne pas entendre les ambiances. Nous négligeons ici ce point (qui a des incidences linguistiques minimes) mais notons que ce trait est autonome du canal. Certes il est exclu de [+écrit] mais il n’apparait pas obligatoirement à la télé ou à la radio.
} 
leur valeur + /- est inscrite (par exemple les CS sont toujours «-interaction »), sinon ils adoptent ou non le trait ${ }^{29}$. Certains aspects contenus dans la représentation ci-dessous seront rencontrés et expliqués plus loin, par exemple la représentation encadrée en majuscules des figures génériques méronymes. En effet, une identification rigoureuse des générèmes conduit à affiner régulièrement les critères externes qui influent sur la structure linguistique. Le déploiement des traits recueillis ci-dessus (figure 8) par l'ensemble des sous-genres n'est pas l'étape ultime conduisant à l'arborescence des générèmes car leur concrétisation permet d'envisager des degrés de granularité plus fins, comme cela sera le cas du trait [+/-mouvement] dépendant de l'image.

(9) Structure de traits du genre de discours Commentaire Sportif

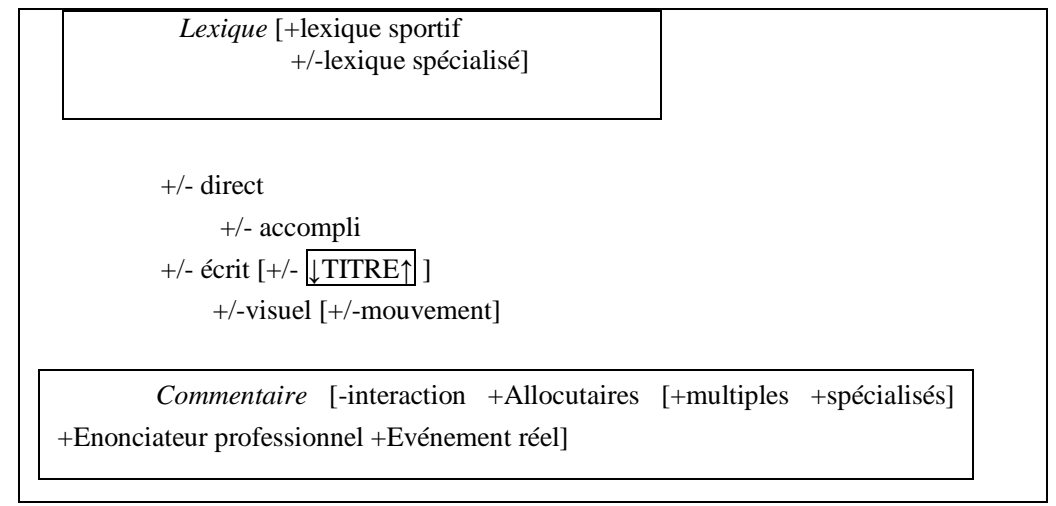

\subsection{Les générèmes du commentaire sportif}

L'avantage d'une structure de traits comme elle est ici conçue ne se limite pas à la mise en relation dans les sous-genres entre faits de langue et critères externes. Un autre intérêt réside dans la dynamique permise par l'exploration de toutes les possibilités générées ${ }^{30}$ par la combinaison des traits oppositifs. Si nous combinons l'ensemble des traits variables principaux (+/-direct, +/-accompli, +/-écrit, +/-visuel), nous obtenons seize combinaisons que nous examinerons. Elles ont toutes en commun les modules lexicaux et commentaires, que nous noterons «CS» par commodité (CS = lexique + Commentaire). Ces combinaisons posent un éclairage sur des commentaires non lexicalisés qui existent, soit de façon autonome soit de façon méronymique. De plus, l'approche systématique impliquée par la structure de traits facilite la recherche de granularité fine des relations entre faits de grammaire et faits externes.

$$
\mathrm{A} /[\mathrm{CS}+\text { écrit }+ \text { visuel }+ \text { direct }+ \text { acc }]
$$

\footnotetext{
${ }^{29}$ Notons toutefois que du temps de la TV noir et blanc, cet élément était intégré par les commentateurs.

30 Le terme « générème » est donc à double sens (et choisi à cette fin) : (a) unité minimale de genre opposable à un autre générème par un et un seul élément grammatical et (b) unités minimales susceptibles d'être générées par la combinaison des traits externes affectant un genre de discours.
} 
On trouve parfois des commentaires d'actions détaillées photo après photo (ou avec des arrêts sur image lors d'émissions TV spécialisées) avec une impression de direct (comparable à une bande dessinée ou un roman-photo). Ce générème est rare aujourd'hui mais était courant dans la presse sportive au début du vingtième siècle. Techniquement la question se pose de distinguer, dans le trait [+visuel], l'opposition [+/-mouvement]. Elle se justifiera si elle s'accompagne de différences linguistiques. Ces dernières restent à identifier, faute de corpus conséquent pour les deux situations de A. Nous en reparlerons plus loin.

$$
\mathrm{B} /[\mathrm{CS}+\text { écrit + visuel + direct + direct'] }
$$

Ce générème est concrétisé par les CS en direct sous-titrés pour malentendants disponibles avec une touche adaptée de la télécommande. Ces sous-titrages suivent au mieux ce qui est effectivement dit par les commentateurs, tout en faisant appel à des didascalies pour décrire les ambiances (applaudissements, huées du public). Les couleurs distinguent les commentaires des didascalies.

$$
\text { C/ [CS +écrit + visuel -direct }+ \text { acc }]
$$

Cela correspondrait aux commentaires nettement différés pour malentendants (sur le modèle de $\mathrm{K}$ ) et aux photos avec légende dans les articles de presse (fréquents dans la première moitié du vingtième). Cette distinction confirme le trait $[+/-$ mouvement] appelé par le trait [+visuel]. La description de l'espace fournit une distinction : la presse écrite utilise pleinement «à gauche, à droite, au centre » ce qui est rarissime ${ }^{31}$ à la télévision car la caméra bouge en fonction de l'action, ce qui semble bloquer un repérage déictique à partir d'un point mobile ${ }^{32}$. De ce fait, la configuration C générée par la structure générale des CS est «seulement» une Figure Générique Intermédiaire (FGI), laquelle réécrit deux générèmes $C^{\prime}$ et $C^{\prime}$ par l'insertion du trait différentiel [+/-mouvement].

$$
\begin{aligned}
& C^{\prime}=\text { Différé malentendant }: /[C S+e ́ c r i t+\text { visuel }[+ \text { mouvement }]-\text { direct }+ \text { acc }] \\
& \left.C^{\prime \prime}=\text { Commentaire photo }: /[C S+e ́ c r i t+\text { visuel [-mouvement }]-\text { direct }+ \text { acc }\right]
\end{aligned}
$$

Il nous faut également revenir à la configuration A. La pertinence d'un trait [mouvement] apparaissant, nous tiendrons A pour une FGI, laquelle produit deux générèmes $A^{\prime}$ avec [-mouvement] et $A$ " [+mouvement].

Le trait [+/-mouvement] est également remarquable d'un point de vue méthodologique. En effet, il n'est déductible intuitivement d'aucun sous-genre (donc lexicalisé). Il résulte de la combinaison des traits appliqués à la réalité empirique des commentaires.

$\mathrm{D} /[\mathrm{CS}+$ écrit + visuel -direct - acc $]$

\footnotetext{
31 Les vieux reporters sportifs disent souvent avec humour et nostalgie: «Autrefois on disait: l'équipe de France est à gauche de votre transistor ».

32 Notons toutefois que l'évolution technique permet des plans fixes captant la totalité de la scène (par exemple la natation au J.O de Londres) assimilables à des images fixes. Les déictiques «à gauche, à droite » réapparaissent.
} 
Cette structure s'applique au commentaire pour malentendants d'une action immédiatement en différé. Le trait [-mouvement] n’est pas opératoire ici. Il est d'ailleurs (quasiment) acquis que ce trait n'est activé que par la réalisation [+accompli].

$$
\mathrm{E} /[\mathrm{CS}+\text { écrit -visuel + direct }+ \text { acc }]
$$

Ce générème réalise les articles de presse commentant un événement sportif avec un effet de direct, une narration à suspens. Ces articles étaient plus nombreux avant 1950 qu'en 2013, où ce générème est plutôt méronyme d'un article du type G, car aujourd'hui la diffusion des informations est nettement plus rapide et répandue. Avant 1950, un lecteur pouvait découvrir l'événement en le lisant, ce n'est plus le cas aujourd'hui.

$$
\mathrm{F} /[\mathrm{CS}+\text { écrit -visuel + direct }+ \text { direct'] }
$$

Avec ce générème, nous entrons dans les commentaires en direct d'internet, genre nouveau inimaginable il y a quinze ans. Les genres se créent et meurent en fonction des évolutions technologiques. Le cas général des commentaires internet est décrit dans la configuration $\mathrm{H}$. Cependant, à la marge, il existe de vrais (ou presque) directs « écrits » lorsqu'un but est marqué. La séquence «but pour Paris !» (sans «oh» cependant) apparaît, puis quelques instant après, le développement précis est exprimé. Ceci dit, ce générème n'est pas autonome ${ }^{33}$. Il se réalise à l'intérieur du générème qui l'appelle (configuration $\mathrm{H}$ ).

G/ [CS +écrit -visuel -direct + acc $]$

Ce générème correspond au compte-rendu d'un événement sportif dans la presse écrite, sportive ou non. Cependant, comme tout article de presse, il nécessite pour être pleinement décrit que lui soit ajouté un module titre, que nous avions plus haut identifié comme générème méronyme, d'où un encadrement complet, en majuscules TITRE. De plus, puisque le titre est susceptible d'interférer sur le reste du générème (les relations anaphoriques notamment), nous lui affectons des flèches montantes et descendantes représentant ces relations : + /- [TITRE $\uparrow$. On objectera, avec raison, que le titre n'est pas à proprement parler un CS dans notre perspective (figure générique type et non occurrence). Toutefois, plusieurs indices invitent à envisager des possibilités d'interactions entre l'article et le titre ${ }^{34}$.

$$
\mathrm{H} /[\mathrm{CS}+\text { écrit -visuel -direct - acc] }
$$

C'est le commentaire en quasi direct sur internet pratiqué par plusieurs médias. La différence avec l'écrit visible dans les commentaires pour malentendants ${ }^{35}$ réside

\footnotetext{
33 A moins d'imaginer une sorte de tchat?

34 Dans Krazem (2012), nous notons la remarquable fréquence des titres à l'infinitif dans la rubrique sportive.

35 Le commentaire pour malentendant a une caractéristique que nous avons gommée ici. En effet, le module commentaire est légèrement différent car il existe deux énonciateurs : le commentateur et le traducteur. Ce dernier a pour mission de coller autant que possible au commentateur mais on se doute bien qu'il reste des imperfections ou des adaptations, moins cependant que dans les soustitrages de films qui condensent nettement le propos réel (Tomaskiewicz, 1995).
} 
dans l'opposition +/- visuel. Comme nous l'annoncions dans la configuration F cidessus, le générème commentaire par internet intègre un générème méronyme peu sollicité mais observable. Du point de vue de la représentation (il en sera ainsi de tous les genres méronymes), nous conviendrons de noter ce genre comme nous l'avons fait pour les titres.

$$
\begin{aligned}
& \mathrm{H}=[\mathrm{CS}+\text { écrit -visuel -direct }-\mathrm{acc}+/-\downarrow \text { Générème } \mathrm{F} \uparrow] \\
& \mathrm{I} /[\mathrm{CS} \text {-écrit }+ \text { visuel }+ \text { direct }+\mathrm{acc}]
\end{aligned}
$$

Voici les commentaires télévisés en "pseudo direct». Le commentateur parle « comme si » l'événement était en direct mais le téléspectateur sait que l'événement a déjà eu lieu. Ce type de commentaire, rares ${ }^{36}$, ne doit pas être confondu avec un événement enregistré en direct et retransmis plus tard (ce qui revient presque au générème $\mathrm{J}$ qui suit).

$\mathrm{J} /[\mathrm{CS}$-écrit + visuel + direct + direct']

C'est le générème " phare » des CS : le commentaire télé en direct, popularisé en France par Roger Couderc (Rugby), Thierry Roland (football) et Robert Chapatte (cyclisme). Notons que les formes grammaticales employées ont contaminé les générèmes [+écrit] dans leur souci stylistique réaliste. C'est visible sur les phrases averbales, inutilisées dans la presse sportive du début du siècle dernier.

\section{$\mathrm{K} /[\mathrm{CS}$-écrit + visuel -direct + acc]}

Ce sont les commentaires narratifs du type «archives du sport». Les amateurs de cyclisme connaissent bien les courts métrages commentés par Jean-Paul Ollivier. Le style est marqué par des formes épiques et emphatiques. Des équivalents [+visuel mouvement] ont vraisemblablement déjà été produits, par exemple dans une rubrique « souvenir» ou «c'était il y a trente ans » proposée par un journal. Nous n'en avons pas cependant d'attestations précises. Le générème $K$ s'applique également aux commentaires d'événements passés récents qu'on entend en fin de journal télévisé. Il s'agit d'une information passée sans scénographie particulière. Les formes linguistiques s'avèrent alors moins épiques.

\section{L/ [CS -écrit + visuel -direct - acc]}

C’est encore un générème méronyme activable par le générème $\mathrm{J}:$ le commentaire télé en léger différé qu'il soit "replay» (on rediffuse une séquence pertinente à vitesse normale) ou «ralenti» (séquence pertinente à vitesse ralentie), lequel est moins utilisé aujourd'hui. Est-il pertinent de distinguer le «replay» du « ralenti »? Autrement dit, la vitesse joue-t-elle un rôle dans les formes grammaticales ? Assurément : les adresses aux téléspectateurs sont notamment plus nombreuses dans les ralentis. L'interjection «ah» $\mathrm{y}$ trouve également un terrain fertile car son

\footnotetext{
36 On récupère ce générème quand plusieurs événements ont lieu en même temps, par exemple lors des journées de ligue des champions, lorsque le match principal en direct est terminé. Il s'agit de préserver le suspens pour le téléspectateur. De plus, il semble que cette pratique était courante avant la mondiovision. Les journalistes, dans les années 60 (coupe du monde 1962), commentaient sur image après avoir vu le match. Les films étaient envoyés ensuite par avion pour être diffusés le lendemain.
} 
énonciation part toujours d'une présupposition. Ce dernier fait de grammaire permet également de distinguer le ralenti d'un commentaire sur image fixe ${ }^{37}$. Aussi, ce que nous avions annoncé plus haut comme étant un générème est formellement une figure générique intermédiaire appelant un trait [+/-vitesse] produisant les générèmes L' (ralenti) et L" (replay). Parallèlement, la configuration fine du sous-genre de J devient ${ }^{38}$ :

$$
\mathrm{J}=[\mathrm{CS} \text {-écrit }+ \text { visuel }+ \text { direct }+ \text { direct' }+/-\downarrow \text { Figure Générique Intermédiaire } \mathrm{L} \uparrow]
$$

Notons enfin que le «ralenti» et même le «replay» ont une existence lexicale (ce sont donc des sous-genres) que les amateurs de sports reconnaissent immédiatement.

\section{$\mathrm{M} / *[\mathrm{CS}$-écrit -visuel + direct + acc $]$}

Cette combinaison ne se concrétise pas. Il faudrait un commentateur faisant comme si c'était en direct, avec un auditeur qui ne disposerait pas de l'image (contrairement au générème $\mathrm{I}$ ). On pourrait imaginer $\mathrm{M}$ si l'événement était fictif (une improvisation théâtrale ?), mais le CS suppose un événement réel dans son module [commentaire]. La configuration $\mathrm{M}$ est donc un générème impossible (et non potentiel) du fait d'une contradiction avec un élément obligatoire. C'est pourquoi nous faisons précéder ici cette configuration d'un astérisque.

$\mathrm{N} /[$ [CS -écrit -visuel +direct + direct']

C'est le générème historique phare des CS : le commentaire radio en direct dont George Briquet et Eugène Saccomano sont les figures emblématiques en France.

$\mathrm{O} /$ [CS -écrit -visuel -direct + acc]

Cette structure de traits trouve sa place à la rubrique sport d'un journal radiophonique. On peut aussi imaginer, faute de corpus, une narration radiophonique de type épique, comparable à la configuration $\mathrm{K}$.

$\mathrm{P} /[\mathrm{CS}$-écrit -visuel -direct - acc]

Il s'agit des reformulations d'un événement immédiatement après sa réalisation. Ce générème est abondamment attesté lors des multiplexes radios ${ }^{39}$. C'est la manifestation la plus accessible expérimentalement du "présent psychologique étendu ».

\footnotetext{
${ }^{37}$ Nous n'explorerons pas davantage les conséquences de l'insertion d'un trait $[+$ mouvement + /rapide] sur l'ensemble des générèmes visuels. En droit, rien n'empêche par exemple un match nettement accompli montré en ralenti à la fin du journal de 20 heures, mais cela n'arrive pas. Une explication intuitive s'appuie sur le public du 20 heures. N'étant pas spécialisé, il ne serait guère intéressé par les aspects techniques mis en valeur par le ralenti).

38 Autrement dit, et avec une autre perspective : le sous-genre « commentaire télé en direct » contient un générème principal et un générème facultatif.

${ }^{39}$ Le principe d'un multiplexe radio (toutes les grandes radios en proposent un lors des soirées de championnats de foot, parfois de rugby) est le suivant. Des commentateurs sont sur tous les stades et un animateur (lui-même journaliste sportif) donne la parole à chacun à tour de rôle, sauf quand un événement important arrive sur un stade. Le commentateur de ce stade devient alors prioritaire et prend la parole en coupant l'événement qui était suivi en direct, et même les publicités en cours de diffusion!
} 


\subsection{Générèmes et tendances grammaticales}

Le tableau (10) reprend les éléments grammaticaux abordés par l'étude des sous-genres pour les appliquer aux générèmes répondant aux deux paramètres +visuel et +écrit. De nombreuses oppositions linguistiques relèvent de la relation au moment présent mais pas seulement. L'adresse aux téléspectateurs est plus forte dans les situations différées. La différence entre I et J est intéressante parce que l'effort d'imitation du direct est limité à la fois par des considérations normatives (il est plus facile de choisir ses formes linguistiques) et de non-perception de formes spécifiques (oh/élision du sujet pronominal).

(10) Tendances des relations genres/grammaires des générèmes +visuel/-écrit

\begin{tabular}{|l|c|c|c|c|c|}
\hline & I & J & K & L' & L” \\
\hline & $\begin{array}{l}\text { TV } \\
\text { pseudo } \\
\text { direct }\end{array}$ & $\begin{array}{l}\text { TV } \\
\text { direct }\end{array}$ & $\begin{array}{l}\text { TV } \\
\text { archives }\end{array}$ & ralenti & replay \\
\hline Phrase averbale complète & + & + & + & ++ & ++ \\
\hline GN, qui & + & + & + & + & + \\
\hline Nom propre seul & ++ & ++ & & - & \\
\hline Oh ! & + & ++ & - & + & + \\
\hline Avec X, qui & & + & - & - & - \\
\hline Présent actuel & + & + & + & + & + \\
\hline Présent historique & - & - & + & - & - \\
\hline Passé simple & - & - & + & - & - \\
\hline Futur (ultérieur) & - & - & & & - \\
\hline Futur historique & ++ & + & + & + & \\
\hline Aller + inf (futur) & ++ & ++ & & + & + \\
\hline Infinitif libre & - & - & & - & - \\
\hline Adresse allocutaire & ++ & + & & ++ & ++ \\
\hline Elision du sujet & & - & + & & \\
\hline Allongement vocalique & + & + & - & & \\
\hline
\end{tabular}

\section{Conclusion}

Rappelons-le, notre approche ${ }^{40}$ des genres de discours vise à améliorer l'observation pour eux-mêmes des éléments linguistiques, du moins une partie d'entre eux : les faits marqués génériquement (ils sont nombreux) qui ne sont pas appelés par la cohésion ou l'articulation des séquences textuelles. L'application que nous en avons faite sur les commentaires sportifs a montré la très grande variété des conséquences génériques que ce «genre de discours » contient, au point que ses manifestations par

$40 \mathrm{Ou}$ des approches ayant le même objectif (voir ici Adam ou encore Condamines, 2007). Dans Krazem (2012) nous proposons une explication des infinitifs injonctifs et leur comparaison avec l'impératif effectuée à partir des genres de discours où ils sont soit nombreux, soit exclus. 
générèmes (passés, présents, émergents) entrent dans toutes les catégories descriptives de Bronckart (2008), lesquelles, soit dit en passant, évitent le piège lexical dans lequel on risque d'enfermer les études grammaticales sur les genres. Les genres de discours ne sont alors pas seulement un élément du contexte parfois utilisé de façon «Samaritaine » pour reprendre l'expression souriante de Kleiber (2009), ils révèlent une partie des propriétés générales d'un fait, pour peu que ce dernier soit potentiellement génériquement marqué. Nous conclurons en présentant d'autres intérêts qu'une telle approche peut renforcer :

(i) Cette représentation facilite les conditions d'une comparaison fine entre différents genres de discours, appartenant ou non au même domaine culturel ou social. La variation entre "sous-genres" s'observe sur les traits. Ainsi le commentaire TV direct/différé se distingue par l'opposition présent physique étendu/ présent physique, que nous avons notée avec les traits +/-direct et +/-accompli. Cette comparaison, grâce à l'autonomie des traits les uns par rapport aux autres, n'est pas prisonnière du domaine d'activité. Des genres très différents entre eux peuvent présenter une intersection importante de leurs traits. C'est le cas entre les commentaires sportifs, les didascalies, les guides de randonnées ou la transcription des débats parlementaires (Krazem, 2009). Il semble en outre relativement aisé d'imaginer un tel système de représentation pour de nombreux genres de discours. Les procédures, les messages, les panneaux d'information, les annonces,... se prêteraient facilement à être décomposés en modules et en traits oppositifs.

(ii) La représentation en traits est adéquate pour rendre compte de genres de discours n'existant plus (par exemple les actualités sportives cinématographiques), ayant connu une évolution diachronique (évolution du compte-rendu sportif écrit) ou potentiellement existants. Notre système de combinaison a satisfait toutes les possibilités théoriques (sauf une) mais la même entreprise se serait heurtée à l'inexistence des commentaires internet il y a dix ans ou encore à la nouveauté des commentaires pour malentendants.

(iii) Une structure de traits n'est pas figée. Elle intègre des traits nouveaux ou émergents. Nous avons, pendant notre redéploiement des combinaisons, noté que le trait [+/-mouvement] avait un impact sur les formes grammaticales utilisées. De même, l'interactivité, a priori exclue des commentaires sportifs, mais qui commence à apparaître, aura des conséquences linguistiques que nous ne mesurons pas encore ${ }^{41}$.

(iv) La structure de trait intègre naturellement les genres ou générèmes méronymes, bien qu'il reste à affiner une représentation anticipant les relations linguistiques entre les genres méronymes et le reste des composants d'un générème.

(v) La recherche des générèmes (ou au moins une démarche qui en intègre les objectifs) est selon nous un préalable à toute entreprise statistique grammaticale sur corpus. Nous suivons totalement Adam (2012) lorsqu'il établit des calculs de fréquence sur les séquences et non exclusivement sur la globalité du genre. Soit dit en passant, on peut s'interroger sur l'intérêt de statistiques portant sur des éléments grammaticaux qui ne seraient pas circonscrites par des générèmes ou des figures

\footnotetext{
${ }^{41}$ Hélène Fretel a attiré notre attention sur les sites internet espagnols (par exemple El Marca.es) où l'interaction entre le commentaire internet et les réactions des internautes qui suivent le match est réelle.
} 
génériques plus grandes neutralisant des variables. Ainsi, lorsqu'on ne distingue que «commentaires sportifs» sans préciser duquel il s'agit, le lecteur, s'il se contente des chiffres, peut penser que le passé simple est un temps légitime. Ce qui n’est ni vrai ni faux mais surtout, ce qui ne fait avancer la compréhension, ni des commentaires sportifs, ni du passé simple.

\section{BIBLIOGRAPHIE}

ADAM, J.M., 1999, Linguistique textuelle, des genres de discours aux textes, Nathan.

ADAM, J.M., 2001, «Types de textes ou genres de discours? Comment classer les textes qui disent de et comment faire ?", Langages n 141, Paris, Larousse, p. 10-27.

Adam, J.M., et HeIDMAnN, U., 2009, le texte littéraire, pour une approche interdisciplinaire, Louvainla-Neuve, Academia Bruylant.

ADAM, J.M., 2010, Les textes : types et protoyypes, Armand Colin.

ADAM, J.M., 2012, «Grammaire, généricité et textualité dans les contes de Perrault : l'exemple de la place de l'adjectif dans le groupe nominal » dans Quand les genres de discours provoquent la grammaire, C. Despierres et M. Krazem (eds.), Lambert Lucas, p. 9-26.

BAKHTine, M., 1984, «Les genres de discours » dans Esthétique de la création verbale, Paris, Gallimard.

BLANCHE-BENVEnISTE, Cl., 1999, «Constitution et exploitation d'un grand corpus », RFLA volume IV fascicule 1., p. 65-74.

BRANCA, S., 1999, «Types, modes et genres : entre langue et discours », Langage et Société, n87, p. $5-24$.

BRES, J., 2007, « Le discours médiatique sportif aux prises avec le temps verbal », in Le français parlé dans les médias, Actes du colloque de l'Université de Stockholm, 8-12 juin 2005, M. Broth, M. Forsgren, C. Norén et F. Sullet-Nylander (éd.), Acta Universitatis stockholmiensis, 83-96.

BIBER, D., 1988, Variation across speach and writing, Cambridge University Press.

BOUQUET, S., 2004, « Sémiotique grammaticale et sémantique des genres de jeux de langage : les pronoms personnels clitiques en français ", Langages $n^{\circ} 153$, p. 28-40.

BOUQUET, S., 2007, «Contribution à une linguistique neo-saussurienne des genres de la parole : une grammaire du morphème $\mathrm{ON} », \operatorname{LINX} \mathrm{n}^{\circ} 56$, p. 143-156.

BRONCKART, J.-P., 2008, «Genres de textes, types de discours, et "degrés" de langue », revue Texto [En ligne], Volume XIII - n $1 / 2$ (2008).

CONDAmines, A., 2007, «L'interprétation en sémantique de corpus : le cas de la construction de terminologies », Revue Française de Linguistique Appliquée, volume XII-1, p. 39-52.

Deulofeu, J., 2000, «Les commentaires sportifs constituent-ils un genre au sens linguistique du terme ? ", Corpus. Méthodologie et applications linguistiques, dirigé par M. Bilger, Paris, Champion, p. 271-295.

Donguy, J., 2007, Poésies expérimentales. Zones numériques, Les presses du réel.

GrinshPun, Y. et KRAZEM, M., 2005, «Quelques marqueurs linguistiques liés au présent de l'indicatif dans les commentaires sportifs en direct», dans C. Despierres et M. Krazem, Du présent de l'indicatif, Dijon, Presses Universitaires de Dijon, p. 117-142. 
GUÉRIN, O., 2011, Nomination et catégorisation des realia exotiques dans les récits de voyage, thèse de doctorat, Paris 3.

Kerbrat-OrReChioni, C., 1995, «Introduction » dans Le trilogue, C. Kerbrat et C. Plantin eds, PUL, p. 17-32.

Kerbrat, C. et Traverso, V., 2004, «Types d'interactions et genres de l'oral », Langages, nº153.

KLEIBER, G., 2009, «D'un contexte à l'autre : aspects et dimensions du contexte », Information Grammaticale, n¹23.

Krazem, M., 2004, «La phrase averbale dans les didascalies » in Verbum, tome XXVI, n³4, p. $355-372$.

Krazem, M., 2005, «le présent sportif » Du présent de l'indicatif Despierres C. et Krazem M. éds. Centre Bachelard, Dijon, p. 77-117.

KRAZEM, M., 2009, «Les marques linguistiques du commentaire didascalique » dans La lettre et la scène : linguistique et sémiologie du texte de théatre, C. Despierres, H. Bismuth, M. Krazem et C. Narjoux, Dijon, Editions Universitaires de Dijon, p. 123-136.

KraZEM, M., 2011, «Zidane, qui passe à Sganarelle, qui lance Platini...ou comment des noms propres deviennent phrases averbales » dans Les énoncés averbaux autonomes entre grammaire et discours, F. Lefeuvre et I. Behr éds., Paris, Ophrys, p. 121-136.

KRAZEM, M., 2012, «Décrire l’infinitif par les genres du discours» dans Quand les genres de discours provoquent la grammaire, C. Despierres et M. Krazem eds., Lambert Lucas, p. 143-180.

Maingueneau, D., 2004, « retour sur une catégorie le genre » dans Adam J.M, Grize J.B et Ali Bouacha M. Texte et discours : catégorie pour l'analyse EUD Dijon, p. 107-118.

Maingueneau, D., 2007, Analyser les textes de communication, Paris, Armand Colin.

MaingueneAu, D., 2012, Les phrases sans texte, Armand Colin

MAlrieux, D. et RASTIER, F., 2001, «Genres et variations morphosyntaxiques », Traitement Automatique des Langues, vol. $42, \mathrm{n}^{\circ} 2$, p. 548-577.

Martin, J. et Rose, D., 2008, Genre relations. Mapping culture, London, Equinox.

LABEAU, E., 2007, «Et un, ou deux, ou trois ? Les temps-champions du compte rendu sportif depuis 1950 ». in: Sémantique et Diachronie du système verbal français. Labeau, Emmanuelle ; Vetters, Carl and Caudal, Patrick (eds), Cahiers Chronos, $n^{\circ} 16$, Amsterdam (NL), Rodopi, p. 203-233.

MATHON, C. et BOUlakia, G., 2011, «Le commentaire sportif en direct : une combinatoire de différentes fonctions de la prosodie ", in Yoo, H-Y et Delais-Roussarie, E. (eds), Actes d'IDP 2009, Paris, 9-11 septembre 2009.

Petitjean, A., 1989, « Les typologies textuelles », Pratiques, 62, p. 86-125.

Rastier F., 2001, Arts et sciences du texte, Paris, PUF.

Tomaskiewicz, T., 1995, Le trilogue, C. Kerbrat et C. Plantin eds, PUL.

WILMET, M., 1980, « Le temps linguistique », L’information grammaticale, nº 7, 1980, p. 6-10. 